\title{
6. Einkommen und Armut in Deutschland: Objektive Einkommenssituation und deren subjektive Bewertung
}

\author{
Constanze Lejeune, Laura Romeu Gordo \& Julia Simonson
}

\section{Kernaussagen}

Das durchschnittliche Einkommen ist zwischen 1996 und 2014 stetig gestiegen, es bestehen aber weiterhin deutliche Unterschiede zwischen Ost- und Westdeutschland: Das reale Einkommen ist unter Berücksichtigung der Preisentwicklung allerdings relativ stabil geblieben. Die Einkommensentwicklung ist seit 1996 für Ost- und Westdeutschland ähnlich. Allerdings liegt das Einkommen in Ostdeutschland in allen Befragungsjahren unter dem Niveau Westdeutschlands.

Das durchschnittliche Einkommen ist zwischen 1996 und 2014 am stärksten für die 60- bis 65-Jährigen gestiegen: Das durchschnittliche Einkommen steigt für alle Altersgruppen. Allerdings ist der Anstieg für die 60- bis 65-Jährigen am deutlichsten. So steigt das durchschnittliche Einkommen für diese Altersgruppe um 77 Prozent und für die anderen Altersgruppen um deutlich weniger als 60 Prozent. Ursache ist vermutlich der Anstieg der Erwerbsbeteiligung in dieser Altersgruppe im gleichen Zeitraum.

Die Bildungsungleichheit bei den Einkommen nimmt zwischen 1996 und 2014 zu: Da die Einkommen der Hochgebildeten stärker gestiegen sind als die der Niedriggebildeten, kommt es zu einer Zunahme der Bildungsungleichheit. Auch die Armutsquote unterscheidet sich zwischen den Bildungsgruppen: Ein Drittel der Personen zwischen 40 und 85 Jahren mit niedrigem Bildungsniveau ist im Jahr 2014 armutsgefährdet. Diese Quote ist seit 1996 stark gestiegen (von 22,2 Prozent auf 34,8 Prozent), während sie für die Hochgebildeten stabil geblieben ist.

Ältere Menschen sind im Vergleich zu anderen Altersgruppen nicht überdurchschnittlich von Armut gefährdet: In Ostdeutschland sind die Armutsquoten der Personen zwischen 70 und 85 (mit 13 Prozent) sogar signifikant niedriger als die Armutsquoten der jüngeren Altersgruppen.

Die Einschätzung der eigenen finanziellen Situation unterscheidet sich zwischen den Bildungsgruppen: Menschen mit niedriger Bildung empfinden sich häufiger auch dann nicht als arm, wenn sie unter die objektive Armutsgrenze fallen. Hochgebildete hingegen empfinden ihr Einkommen häufiger auch dann nicht als ausreichend, wenn es über der objektiven Armutsgrenze liegt.

\subsection{Einleitung}

Die materielle Lage ist von entscheidender Bedeutung für die Lebenssituation im Alter. Ein ausreichendes Einkommen ist eine grundlegende Voraussetzung für gesellschaftliche Teil- habe und Integration aller - und damit auch älterer - Menschen. Aus wirtschaftlicher und sozialpolitischer Sicht ist es von hoher Bedeutung, wie die materielle Lage in der Population 
älterer Menschen verteilt ist und wie sich diese Verteilung entwickelt hat. Hat sich die materielle Lage älterer Menschen in den letzten Jahren verbessert oder verschlechtert? Gibt es bestimmte gesellschaftliche Gruppen, bei denen ein besonderer materieller Bedarf festzustellen ist? Im vorliegenden Kapitel steht das Einkommen älterer Menschen im Mittelpunkt (das darauffolgende Kapitel 7 ist dem Thema Vermögen gewidmet). Von besonderer Bedeutung bei der Betrachtung der materiellen Lage ist das Vorliegen von Armut im Alter: Lebt ein älterer Mensch in Armut, ist es wenig wahrscheinlich, dass er sich aus eigener Kraft aus dieser Situation befreien kann. Daher wird die Verteilung und Entwicklung von Altersarmut in diesem Kapitel eingehend betrachtet. Zudem ist die Frage, ob die materielle Lage in unterschiedlichen gesellschaftlichen Gruppen unterschiedlich verteilt ist, für die Sozialpolitik von besonderer Bedeutung, weil Maßnahmen zur Überwindung dieser Ungleichheit und Armut für unterschiedliche gesellschaftliche Gruppen verschieden ausfallen müssen. Daher werden im Folgenden Frauen und Männer, Menschen in Ost- und Westdeutschland sowie Menschen, die unterschiedlichen Bildungsgruppen angehören, im Vergleich betrachtet.

Das Einkommen setzt sich aus den Einnahmen zusammen, die eine Person in regelmäßigen Abständen erhält, etwa in Form der Entlohnung einer Arbeit oder in Form von Rentenbezügen. Die Quellen (und die Höhe) des Einkommens verändern sich im Lebensverlauf. Im Erwerbsalter ist die Hauptquelle des regelmäßigen Einkommens das Erwerbseinkommen. Dagegen setzt sich das Einkommen im Ruhestandsalter überwiegend aus Renteneinkünften (insbesondere der Gesetzlichen Rentenversicherung) zusammen und liegt meist unter dem vormaligen Einkommensniveau aus Erwerbseinkünften. Es ist für verschiedene Seiten von Interesse, wie hoch das Einkommensniveau der Personen im Rentenbezug ist und wie es sich im Zeitvergleich gewandelt hat. Auf der wirtschaftlichen Seite ist das Einkommen ein wichtiger Konsumdeterminant. Schließlich ist die Kenntnis der Einkommensverteilung zwischen den verschiedene Gruppen als Basis für die Sozial- politik von besonderer Bedeutung. Das verfügbare Einkommen ist in Deutschland insgesamt seit Anfang der 2000er Jahre stetig gestiegen. Allerdings erfolgt dieser Anstieg nicht für alle Einkommensgruppen gleichermaßen. Am meisten haben höhere Einkommensgruppen von der Steigerung profitiert, sodass die Ungleichheit in der Gesamtbevölkerung in dieser Periode angestiegen ist (Goebel, Grabka, \& Schröder 2015).

Ein in der politischen und wissenschaftlichen Diskussion zunehmend relevantes Thema ist das der Altersarmut. Während absolute Armut die mangelnde Befriedigung von Grundbedürfnissen ist, wird ,relative Armut' als Mangel im Vergleich $\mathrm{zu}$ gesellschaftlich festgelegten Einkommensgrenzen definiert. Die (relative) Armutsgrenze liegt in Deutschland bei 60 Prozent des mittleren Nettoäquivalenzeinkommens. Obwohl ältere Menschen im Vergleich zu anderen gesellschaftlichen Gruppen auch heute noch nicht überdurchschnittlich von Armut gefährdet sind (Vogel \& Künemund 2014), ist mit einem Anstieg der Armutsgefährdung für Personen im Rentenbezug zu rechnen. Das zeigt sich vor allem daran, dass die geburtenstarken Jahrgänge (Babyboomer) erst noch in den Rentenbezug kommen und sich bei ihnen durch diskontinuierliche Erwerbsverläufe bereits jetzt ein erhöhtes Risiko zukünftiger Altersarmut abzeichnet (Simonson 2013; Simonson, Kelle, Romeu Gordo, Grabka, Rasner, \& Westermeier 2012). Besonders stark davon betroffen sind alleinlebende Frauen im Rentenalter, da sich ihre Lebensverläufe durch brüchige Erwerbsverläufe und niedrige Einkommensniveaus auszeichnen und ihre niedrigen Renteneinkommen im Fall der Verwitwung im Alter nicht mehr durch einen Partner aufgefangen werden können (Groh-Samberg 2009). Insbesondere bei der Betrachtung von Altersarmut ist daher der Vergleich zwischen Geschlechtern wichtig, da sich unterschiedliche typische Erwerbsverläufe in einem erhöhten Armutsrisiko für Frauen kumulieren. Allerdings zeichnet sich ab, dass zukünftig auch Männer aufgrund von diskontinuierlichen Erwerbsverläufen ein erhöhtes Risiko der Altersarmut aufweisen werden (Simonson et al. 2012). Dieser Befund gilt insbesondere für Ostdeutschland. Dort ist die Armutsquote 
in der Gesamtbevölkerung fast doppelt so hoch wie in Westdeutschland (Grabka, Goebel, \& Schupp 2012). Auch in Rentnerhaushalten liegt die Armutsquote in Ostdeutschland, je nach Berechnungsgrundlage, deutlich über dem Niveau in Westdeutschland (Noll \& Weick 2013; Zhu, Weißenborn, \& Buscher 2011).

Neben objektiven Armutsindikatoren sind in der Diskussion über Altersarmut auch subjektive Indikatoren von Relevanz. Die subjektive Bewertung der eigenen Einkommensposition kann manchmal sogar wichtiger sein als der Sachverhalt an sich. Denn die Wahrnehmung von sozialer Ungleichheit und eingeschränkten Handlungsoptionen bestimmt das Verhalten von Menschen häufig stärker als der universelle Vergleich mit allen anderen Gesellschaftsmitgliedern, dem objektive Messungen relativer Armut zugrunde liegen. Dabei kann es zu bedeutsamen Abweichungen zwischen objektiv und subjektiv gemessener Armut kommen: Wenn sich Menschen, die objektiv als arm zu bezeichnen sind, selbst nicht als arm einschätzen, liegt eine subjektive Unterschätzung der eigenen Bedürftigkeit vor. Schröder (2013) zeigt, dass der Anteil der Bevölkerung, der einschätzt, dass ihr Haushaltseinkommen deutlich unter dem nach eigenem Maßstab notwendigen Mindestniveau liegt, niedriger ist als der Anteil der Personen, die als ,relativ arm' gelten, da ihr Haushaltseinkommen weniger als 60 Prozent des Medians der Gesamtbevölkerung beträgt. Die subjektiv empfundene Armut ist in Deutschland demnach weniger ausgeprägt als die (relative) objektive Armut. Das nach eigener Einschätzung zum Leben mindestens notwendige Einkommen kann nach der jeweiligen Lebenslage variieren. Von besonderem Interesse ist daher, diese subjektive Bedarfswahrnehmung nach Lebenssituation differenziert zu untersuchen.

Im vorliegenden Kapitel wird die Entwicklung der Einkommen sowie der objektiven und subjektiven Armut von Personen im Alter ab 40 Jahren im Hinblick auf Alters-, Regions- und Bildungsunterschiede betrachtet.

Es werden die folgenden Fragen untersucht:

1. Wie verteilt sich das durchschnittliche monatliche Haushaltseinkommen und wie hat sich diese Verteilung seit 1996 gewandelt? Welche Unterschiede zwischen gesellschaftlichen Gruppen sind zu finden?

2. Wie verteilt sich Armut in der zweiten Lebenshälfte und wie hat sich diese Verteilung seit 1996 gewandelt? Welche Unterschiede zwischen gesellschaftlichen Gruppen sind zu finden?

3. Unterscheiden sich objektive von subjektiven Armutsquoten? Welche Unterschiede zwischen gesellschaftlichen Gruppen sind zu finden?

\subsection{Datengrundlage ${ }^{1}$}

Daten. Als Datengrundlage dienen die Informationen von allen 40- bis 85 -jährigen Befragten der Basisstichproben 1996, 2002, 2008 und 2014 des Deutschen Alterssurveys (DEAS), die am mündlichen Interview teilgenommen haben.

Einkommen. Die hauptsächlich genutzte Haushaltseinkommen-Variable setzt sich aus

1 Die Daten des DEAS können für wissenschaftliche Zwecke kostenlos beim Forschungsdatenzentrum des DZA (www.fdz-dza.de) bezogen werden. der Summe der von allen Mitgliedern eines Haushaltes erzielten persönlichen Nettoeinkommen und der nicht differenziert einzelnen Mitgliedern zuzurechnenden Einkommenskomponenten zusammen. Das Einkommen der Befragten wird im mündlichen Interview erfasst. In der schriftlichen Erhebung des DEAS wird auch die Zusammensetzung des Haushaltseinkommens erfragt. Diese Information wird verwendet, um fehlende Angaben aus dem mündlichen Interview zu ergänzen. Es erfolgt 
eine Äquivalenzgewichtung des Einkommens auf Basis der Angaben zur Haushaltszusammensetzung um die Bedarfe unterschiedlicher Haushaltstypen zu berücksichtigen. Die Summe aller Einkünfte wird nicht durch die einfache Anzahl der Mitglieder eines Haushalts, sondern durch eine gewichtete Summe der Mitglieder der Bedarfsgemeinschaft geteilt (Statistisches Bundesamt 2015b). Für die Berechnung der Äquivalenzeinkommen wird die neue OECDSkala verwendet, bei welcher der Hauptbezieher beziehungsweise die Hauptbezieherin des Einkommens mit dem Faktor 1,0 in die Gewichtung eingeht, Mitglieder der Bedarfsgemeinschaft im Alter von 14 und mehr Jahren mit 0,5 und die restlichen Mitglieder mit 0,3. Für die Angaben zum preisbereinigten Einkommen dient das Jahr 1996 als Referenz, das heißt, die Ergebnisse werden in Preisen von 1996 ausgedrückt. Damit kann die Entwicklung des Einkommens unter Berücksichtigung der Preisentwicklung zwischen den Erhebungsjahren beobachtet werden.

Objektive Armut. Als objektiver Armutsindikator wird die relative Einkommensarmut verwendet. Armut liegt nach dieser Definition dann vor, wenn eine Person weniger als 60 Prozent des gesellschaftlichen Medianeinkommens zur Verfügung hat. Wir berichten über den Anteil der Population, der sich unter diese Grenze befindet. ${ }^{2}$ Die Ermittlung der Armutsgrenze erfolgt auf Basis des Sozio-oekonomischen Panels (SOEP), das auf einer repräsentativen Stichprobe der Gesamtbevölkerung basiert.

Ungleichheit: Als Ungleichheitsmaß werden Gini-Koeffizienten berechnet. Der Gini-Koeffizient ist ein Maß zur Quantifizierung der relativen Konzentration einer Einkommensverteilung. Im Falle der maximalen Gleichverteilung der Einkommen (d. h. jede Person bezieht exakt das Durchschnittseinkommen der betrachteten Grundgesamtheit) nimmt der Gini-Koeffizient den Wert Null an, während er bei einer maximal ungleichen Einkommensverteilung (d. h. eine einzige Person bezieht das komplette Einkom- men der betrachteten Grundgesamtheit für sich alleine) den Wert Eins annimmt.

Subjektive Armut. Neben der objektiven Armutsgrenze gibt es das Konzept der subjektiven Armutsgrenzen (,social subjective poverty line'). Diese subjektive Armutsgrenze wird ermittelt, indem der Betrag abgefragt wird, den die Befragten als niedrigstes Nettoeinkommen angeben, mit dem ihr Haushalt finanziell zurechtkäme. Diese subjektive Armutsgrenze wird genutzt, um eine Betroffenheit von Armut anhand der objektiven Armutsgrenze mit der subjektiven Wahrnehmung zu vergleichen (Ravallion 2012).

Gruppierungsvariablen. Zur Prüfung von Altersunterschieden wurden - analog zur Schichtung der Stichprobe - drei Altersgruppen verwendet: 40 bis 54 Jahre, 55 bis 69 Jahre und 70 bis 85 Jahre. Altersunterschiede im Wandel wurden anhand von Sechs-Jahres-Altersgruppen untersucht, um Überschneidungen zwischen Erhebungszeitpunkten und Altersgruppen zu vermeiden ( 42 bis 47 Jahre, 48 bis 53 Jahre, 54 bis 59 Jahre, 60 bis 65 Jahre, 66 bis 71 Jahre, 72 bis 77 Jahre, 78 bis 83 Jahre).

Weiterhin wurden Geschlechterunterschiede und Regionsunterschiede (Ost-/Westdeutschland) untersucht.

Zur Untersuchung von Bildungsunterschieden wurden - basierend auf einer reduzierten ISCED-Klassifizierung - drei Bildungsgruppen unterschieden: Personen mit niedriger, mittlerer und hoher Bildung (vgl. Kapitel 2).

Analyse. In den Abbildungen und im Text sind gewichtete prozentuale Verteilungen angegeben. Um Unterschiede zwischen Gruppen und/oder Jahren auf statistische Signifikanz $(p<, 05)$ zu testen, wurden in Abhängigkeit vom Skalenniveau logistische Regressionen oder Kovarianzanalysen berechnet, wobei für die Stratifizierungsvariablen Altersgruppe, Geschlecht und Region (Ost-/Westdeutschland) kontrolliert wurde. Das genaue Vorgehen ist in Kapitel 2 beschrieben.

2 In der amtlichen Statistik wird diese Quote in der Regel als Armutsgefährdungsquote bezeichnet. 


\subsection{Entwicklung der monatlichen Haushaltseinkommen im Zeitvergleich}

Einkommen zusammen mit Vermögen (vgl. Kapitel 7) sind ein wesentlicher Aspekt der materiellen Ressourcen im Alter. Im Folgenden wird untersucht, wie sich das verfügbare Einkommen für Menschen zwischen 40 und 85 Jahren seit 1996 entwickelt hat und ob es dabei Unterschiede zwischen verschiedenen Personengruppen gibt.

\section{Das durchschnittliche Einkommen ist zwischen 1996 und 2014 stetig gestiegen, es bestehen aber weiterhin deutliche Unterschiede zwischen Ost- und Westdeutschland.}

Das durchschnittliche Nettoäquivalenzeinkommen der Menschen ab 40 Jahren in Deutschland im Jahr 2014 beträgt 1.922 Euro (vgl. Tabelle A 6-1 im Anhang). Dieser Wert ist leicht höher als der Gesamtpopulationsdurchschnitt von 1.872 Euro (Statistisches Bundesamt 2015b; Wert für 2013). Darüber hinaus ist dieser Betrag seit 1996 stetig gestiegen: Im Jahr 1996 lag das durchschnittliche Nettoäquivalenzeinkommen bei 1.283 Euro, 2002 bei 1.527 Euro und 2008 bei 1.684 Euro. Bei Berücksichtigung der Preisentwicklung relativiert sich die steigende Tendenz. Besonders zwischen 2002 und 2014 ist das durchschnittliche reale Einkommen kaum, das heißt um lediglich circa fünf Prozent (von
1.407 Euro auf 1.471 Euro), gestiegen (vgl. Tabelle A 6-2 im Anhang). Auch Goebel et al. (2015) berichten einen Anstieg von fünf Prozent für die gesamte Population zwischen 2000 und 2012. Das heißt, obwohl das Einkommen seit 1996 gestiegen ist, ist die Kaufkraft aufgrund der Preisentwicklung relativ stabil geblieben.

Um die Entwicklungen für verschiedene Gruppen zu verdeutlichen, werden im Folgenden die Veränderungen der Einkommen in Relation zum gruppenspezifischen durchschnittlichen Einkommen des Jahres 1996 dargestellt.

In Abbildung 6-1 ist dargestellt, wie die Nettoäquivalenzeinkommen über die Erhebungsjahre steigen. Diese Entwicklung ist sowohl für Frauen als auch für Männer zu beobachten, für die das Nettoäquivalenzeinkommen seit 1996 jeweils signifikant steigt. Allerdings ist die Steigerung bei Männern etwas ausgeprägter als bei Frauen. Das durchschnittliche Nettoäquivalenzeinkommen für Männer im Jahr 2014 beträgt 2.015 Euro und für Frauen 1.831 Euro.

Zudem lässt sich eine ähnliche Einkommensentwicklung für Ost und Westdeutschland beobachten. Allerdings liegt das Einkommen in Ostdeutschland in allen Befragungsjahren unter dem Niveau Westdeutschlands. Im Jahr 2014 liegt das durchschnittliche Einkommen in Westdeutschland bei 2.013 Euro, in Ostdeutschland bei 1.525 Euro. 


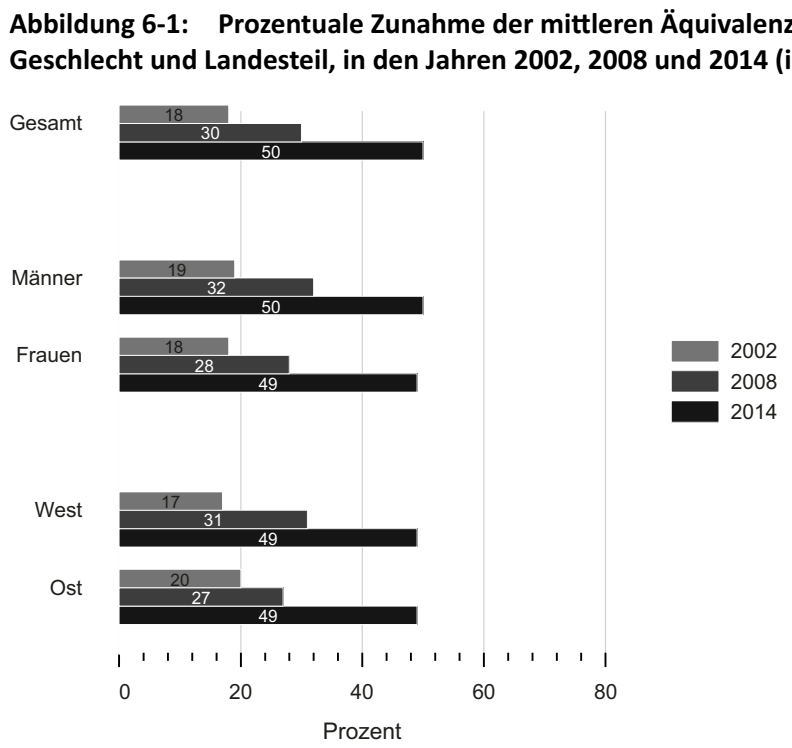

$\overline{\text { Quelle: DEAS } 1996}(n=4.275), 2002(n=2.686), 2008(n=5.445), 2014(n=5.400)$, gewichtet, gerundete Angaben; $(p<, 05)$.

Referenzjahr ist 1996 (=0). Zuwächse zwischen 1996 und 2014 für Gesamt, beide Geschlechter und Landesteile signifikant.

\section{Das durchschnittliche Einkommen ist zwischen 1996 und 2014 am stärksten für die 60- bis 65-Jährigen gestiegen.}

Betrachtet man die Einkommensentwicklung nach Altersgruppen (Abbildung 6-2), erkennt man den gleichen Trend für alle Altersgruppen: Das Nettoäquivalenzeinkommen steigt über die Erhebungsjahre an. Allerdings sind Unterschiede bei dieser Entwicklung zwischen den Altersgruppen zu beobachten. So ist der Anstieg des Einkommens in der Altersgruppe der 60- bis 65-Jährigen höher als in allen anderen Altersgruppen (jünger und älter).
Eine mögliche Erklärung könnte der starke Anstieg der Erwerbspartizipation von Personen zwischen 60 und 65 Jahren seit 1996 sein (vgl. Kapitel 3), sodass diese Gruppe öfter ein Erwerbseinkommen als Hauptverdienstquelle angibt, das in der Regel höher ausfällt als die Rente. Allgemein ist zu beobachten, dass das durchschnittliche Einkommen für Personen im Erwerbsalter höher ist, als für Personen im Rentenalter. So beträgt das durchschnittliche Einkommen im Jahr 2014 für Personen, die jünger als 65 Jahre sind, 2.022 Euro und für Personen im Alter von 65 Jahren alt oder älter 1.738 Euro (ohne Abbildung). 


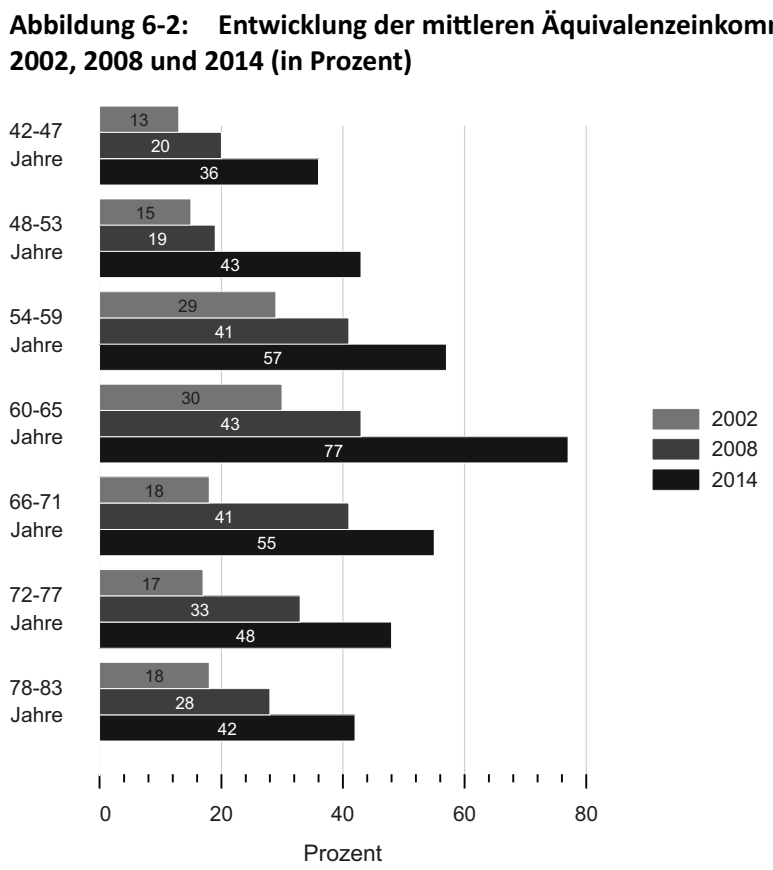

Quelle: DEAS $1996(n=4.009), 2002(n=2.546), 2008(n=5.184), 2014(n=5.179)$, gewichtet, gerundete Angaben; $(p<, 05)$.

Signifikante Zuwächse zwischen 1996 und 2014 für alle Altersgruppen. Interaktion zwischen Erhebungswelle und Altersgruppe signifikant. Deutlichere Zuwächse zwischen 1996 und 2014 für die Altersgruppe 60 bis 65 Jahre.

\section{Die Bildungsungleichheit bei den Einkommen nimmt zwischen 1996 und 2014 zu.}

Die steigende Entwicklung der Einkommen seit 1996 ist für alle Bildungsgruppen zu beobachten (Abbildung 6-3). Allerdings ist der Anstieg bei Personen mit höherer Bildung am stärksten. Für Personen mit hoher Bildung hat sich das durchschnittliche Einkommen von 1.597 Euro im Jahr 1996 auf 2.427 Euro im Jahr 2014 erhöht. Die Einkommen von Personen mit niedriger Bildung sind am wenigsten gestiegen (von 959 Euro im Jahr 1996 auf 1.169 Euro im Jahr 2014). Bei Berücksichtigung der Preisentwicklung kann festgestellt werden, dass die Realeinkommen für Personen mit niedriger Bildung seit 2002 sogar gesunken sind. Dies bedeutet, dass Einkommensunterschiede zwischen den Bildungsgruppen seit 1996 steigen und dadurch 2014 mehr Ungleichheit zwischen den Gruppen zu beobachten ist. 


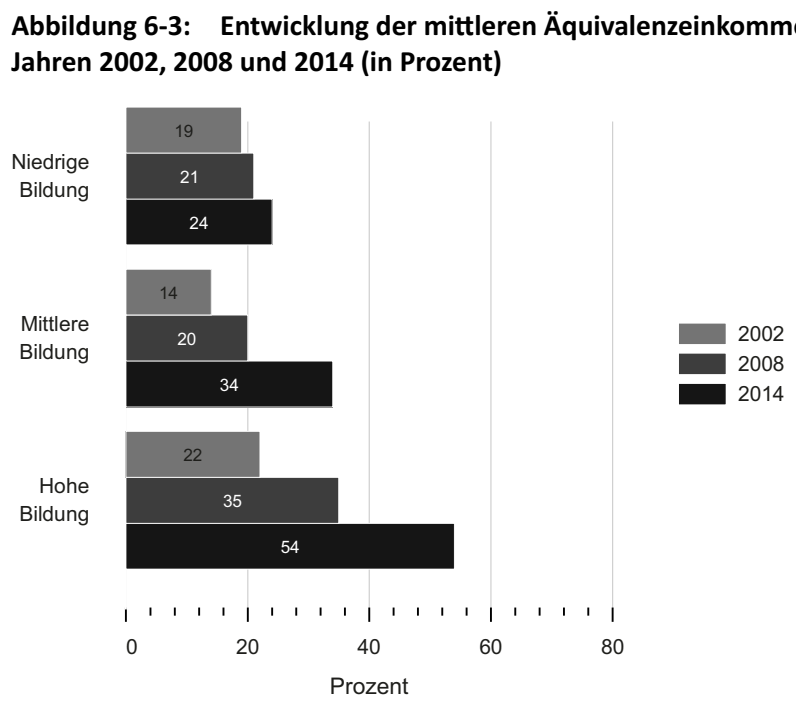

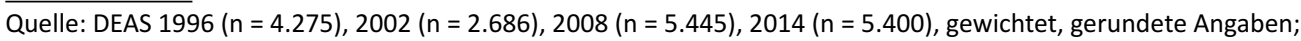
$(p<, 05)$.

Signifikante Zuwächse zwischen 1996 und 2014 für alle Bildungsgruppen. Interaktion zwischen Erhebungsjahr und Bildung signifikant. Deutlichere Zuwächse zwischen 1996 und 2014 für hohe Bildung.

Um die steigende Ungleichheit genauer zu analysieren, wurde der sogenannte Gini-Koeffizient berechnet. $\mathrm{Zu}$ beobachten ist ein leichter Anstieg des Gini-Koeffizienten zwischen 1996 und 2014 (von 0,27 auf 0,30). Der stärkste Anstieg findet zwischen 2002 und 2008 statt. Zwischen 2008 und 2014 bleibt er unverändert. Dies bedeutet, dass die Ungleichheit der Einkommen von Menschen in der zweiten Lebenshälfte in Deutschland seit 1996 zugenommen hat, auch wenn sie in den letzten Jahren relativ stabil geblieben ist. Diese Entwicklung wird auch in anderen Studien beschrieben (zum Beispiel Goebel et al. 2015).

Insgesamt zeigt sich bei der Betrachtung der Einkommen, dass weiterhin starke Un- terschiede zwischen den Geschlechtern sowie zwischen Ost- und Westdeutschland bestehen. Betrachtet man die Einkommen nach verschiedenen Altersgruppen, lässt sich eine relative Verbesserung der Personen zwischen 60 und 65 beobachten, vermutlich durch die steigende Ewerbspartizipation der Personen in dieser Altersgruppe.

Des Weiteren zeigt sich bei Personen mit hoher Bildung ein höherer Anstieg in den Einkommen als bei Personen mit niedrigerer Bildung. Dadurch werden die Unterschiede zwischen den Bildungsgruppen größer. Diese Zunahme der Ungleichheit spiegelt sich auch im Gini-Koeffizienten wider, der zwischen 1996 und 2014 leicht gestiegen ist.

\subsection{Entwicklung der objektiven Armutsquoten}

Um die Ungleichheit von Einkommen bewerten zu können, ist die zusätzliche Betrachtung von Armut notwendig. Diese zeigt an, ob Haushalte unter einem bestimmten Einkommensniveau im Vergleich zur Gesamtbevölkerung leben. Bei der Betrachtung von Armut wird deutlich, dass 
eine ausreichende finanzielle Ausstattung zur sozialen Teilhabe in der Gesellschaft notwendig ist und ein zu geringes Einkommen diese Teilhabe verhindert oder zumindest erschwert.

Die Armutsquote der Menschen in der zweiten Lebenshälfte ist seit 1996 signifikant von 9,7 Prozent auf 12,3 Prozent gestiegen (Abbildung 6-4). Diese Anteile liegen leicht unter den Armutsquoten der amtlichen Statistik, die auf Mikrozensus-Basis berechnet werden: Hier liegen die Quoten im Jahr 2014 für die 50- bis unter 65-Jährigen bei 13,0 Prozent und für die Personen ab 65 Jahren bei 14,4 Prozent (Statistische Ämter des Bundes und der Länder 2015). Werden die Armutsquoten für diese Altersgruppen mit DEAS-Daten berechnen, liegen die Quoten für die 50- bis unter 65 -Jährigen bei 12,5 und für Personen ab 65 Jahren bei 12,7 Prozent. Das heißt, die Abweichung zur amtlichen Statistik ist bei höheren Altersgruppen größer. Das könnte an Selektionseffekten bei älteren Befragten im DEAS liegen. Der bedeutsame Anstieg der Armutsquoten findet zwischen 1996 und 2008 statt, zwischen 2008 und 2014 ist der Anstieg nicht signifikant.
Für Frauen und Männer ist im Blick auf Armut eine ähnliche Entwicklung zu beobachten, wobei die Armutsquoten der Frauen höher sind als die der Männer. Im Jahr 2014 verfügen 13,6 Prozent der Frauen ab 40 Jahren über weniger als 60 Prozent des gesellschaftlichen Medianeinkommens. Für Männer ist dies für nur 10,8 Prozent der Fall.

Auch bei der Armutsquote gibt es einen signifikanten Unterschied zwischen West- und Ostdeutschland: Die ostdeutsche Armutsquote liegt in allen Jahren über der Westdeutschlands. Bei 17,5 Prozent der Ostdeutschen ab 40 Jahren liegt das Einkommen im Jahr 2014 unter der Armutsgrenze, während dies nur für 11,0 Prozent der Westdeutschen der Fall ist. In beiden Regionen sind die Armutsquoten seit 1996 gestiegen. In Ostdeutschland ist ein starker Anstieg zwischen 1996 und $2008 \mathrm{zu}$ beobachten. Zwischen 2008 und 2014 stagniert die Armutsquote für Ostdeutschland auf hohem Niveau. In Westdeutschland ist der Anstieg zwischen 1996 und 2008 nicht signifikant. Zwischen 2008 und 2014 ist hier jedoch ein signifikanter Anstieg von 9,2 Prozent auf 11,0 Prozent festzustellen.

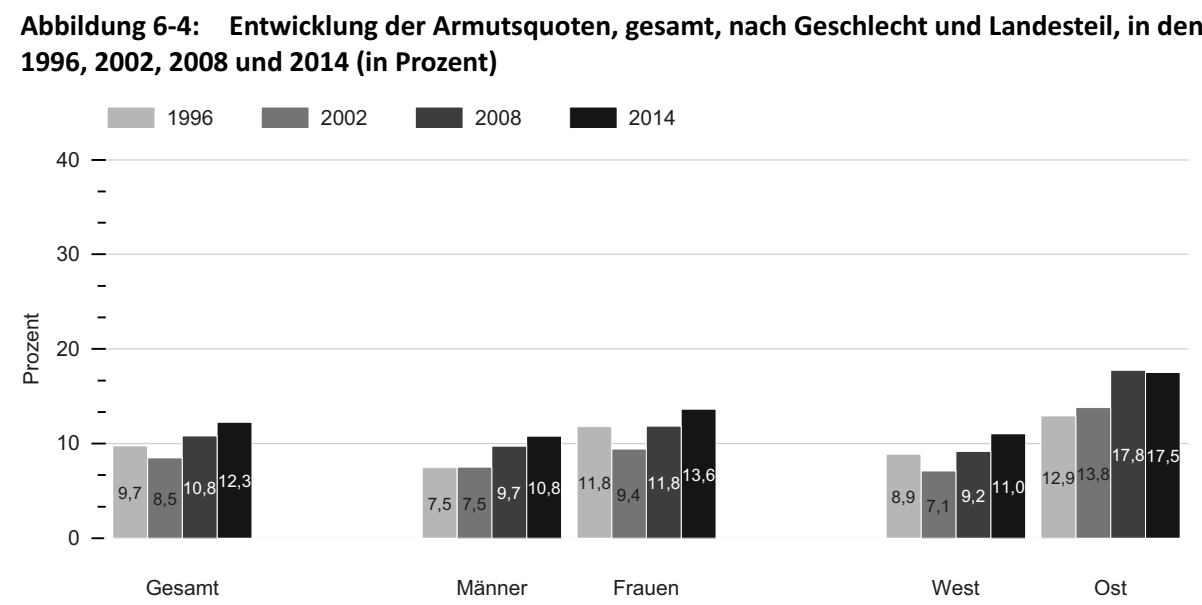

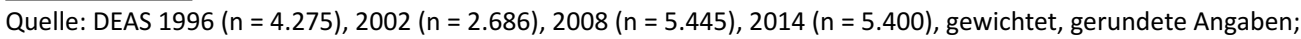
$(p<, 05)$.

Zuwächse zwischen 1996 und 2014 für Gesamt, beide Geschlechter und Landesteile signifikant.

Ältere Menschen sind im Vergleich zu anderen Altersgruppen nicht überdurchschnittlich von Armut gefährdet.
Betrachtet man die Armutsquoten nach Altersgruppen, ist zu erkennen, dass ältere Menschen (ab 70 Jahren) im Vergleich zu anderen Alters- 
gruppen nicht überdurchschnittlich von Armut betroffen sind. In Ostdeutschland sind die Armutsquoten der Personen zwischen 70 und 85 Jahren sogar signifikant niedriger als die Armutsquoten der jüngeren Altersgruppen (vgl. Tabelle A 6-3 im Anhang). Diese Befunde stehen in Einklang mit Ergebnissen auf Mikrozensus-Basis, in denen die Armutsquote für die Gesamtbevölkerung bei 15,4 Prozent liegt und für Personen über 65 Jahren 14,4 Prozent beträgt (Statistische Ämter des Bundes und der Länder 2015). Darüber hinaus ist die Entwicklung der Armutsquoten innerhalb der Altersgruppen ähnlich, sodass sich die relative Position Älterer mit der Zeit nicht verschlechtert hat.

Größere Differenzen in der Entwicklung der Armutsquoten sind zwischen verschiedenen
Bildungsgruppen $\mathrm{zu}$ beobachten (Abbildung 6-5). Die Armutsquote für Personen mit hoher Bildung ist im Jahr 2014 mit 4,5 Prozent im Vergleich zu niedriggebildeten Personen sehr niedrig. Bei den Niedriggebildeten liegen 34,8 Prozent unter der Armutsgrenze. Darüber hinaus hat sich die Armutsquote für Personen mit hoher Bildung seit 1996 nicht signifikant verändert. Demgegenüber ist die Armutsquote der niedriggebildeten Personen in dieser Periode sehr stark, um 12,6 Prozentpunkte (von 22,2 auf 34,8 Prozent), angestiegen. Dieses Ergebnis bestätigt die steigende Ungleichheit zwischen den Bildungsgruppen, die bereits in der Analyse der Entwicklung der durchschnittlichen Einkommen (Abbildung 6-3) zu sehen war.

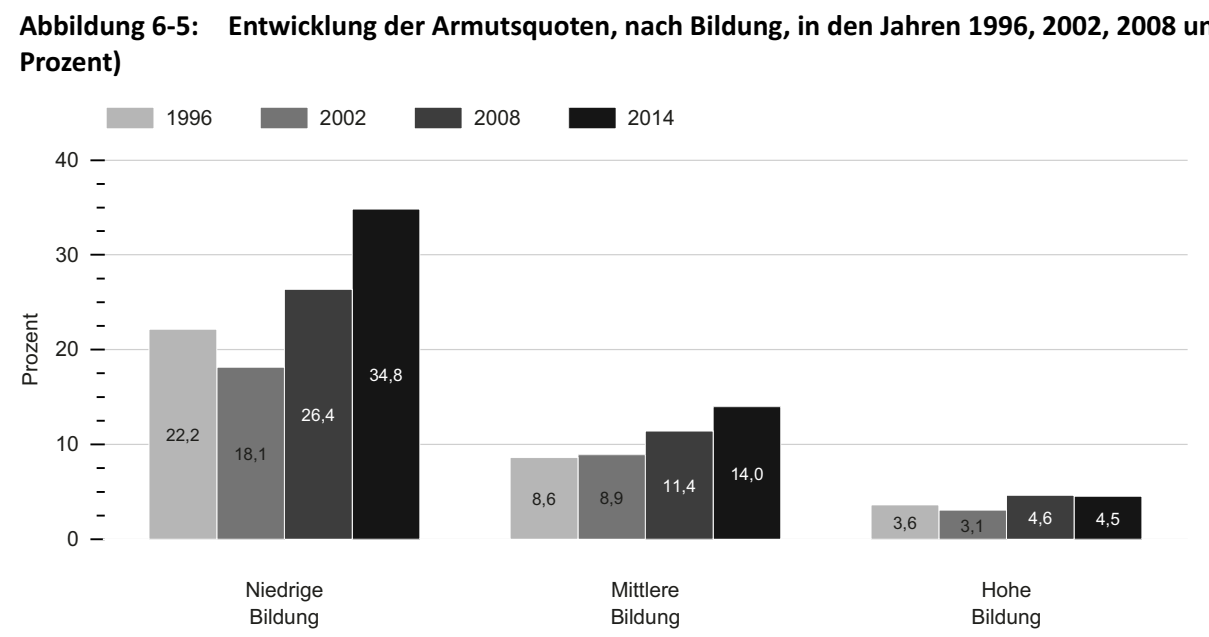

$\overline{\text { Quelle: DEAS } 1996}(n=4.270), 2002(n=2.686), 2008(n=5.445)$ und $2014(n=5.399)$, gewichtet, gerundete Angaben; $(p<, 05)$.

Signifikante Unterschiede zwischen 1996 und 2014 nur für niedrige und mittlere Bildung. 


\subsection{Subjektive Armut und ihr Zusammenhang mit objektiver Armut}

Seit 2014 wird im DEAS durch einen weiteren Indikator Armut gemessen. Dabei wird nach einem Mindesteinkommen gefragt, um finanziell zurecht zu kommen. Es wird also eine subjektive Einkommensgrenze erhoben, unter der man finanziell zu schlecht gestellt ist, um in Deutschland gesellschaftlich teilhaben zu können. Die subjektive Armutsquote umfasst diejenigen, deren Einkommen unterhalb dem liegt, was sie laut eigener Angabe benötigen.

Im Folgenden werden die subjektiven Armutsquoten für unterschiedliche Bevölkerungsgruppen betrachtet und mit den objektiven Armutsquoten verglichen. Auf diese Weise kann geprüft werden, ob es mehr Personen gibt, die sich subjektiv arm fühlen, weil sie nicht genug
Geld haben, um finanziell ,über die Runden zu kommen', als Personen, die nach dem Standard der objektiven Armutsdefinition als arm klassifiziert werden. Darüber hinaus wird untersucht, ob es diesbezüglich einen Effekt im Hinblick auf Alter, Geschlecht, Bildung oder Region gibt.

In Abbildung 6-6 werden die subjektiven Armutsquoten für 2014 dargestellt. Die subjektive Armutsquote aller Menschen in der zweiten Lebenshälfte beträgt 15,0 Prozent. Das bedeutet, dass 15,0 Prozent der Menschen ab 40 Jahren einschätzen, dass ihre Einkommen nicht ausreichen, um finanziell ,über die Runden zu kommen. Diese Quote ist um fast drei Prozentpunkte höher als die objektive Armutsquote von 12,3 Prozent.

Abbildung 6-6: Subjektive Armutsquoten, gesamt, nach Alter, Geschlecht, Bildung und Landesteil, im Jahr 2014 (in Prozent)

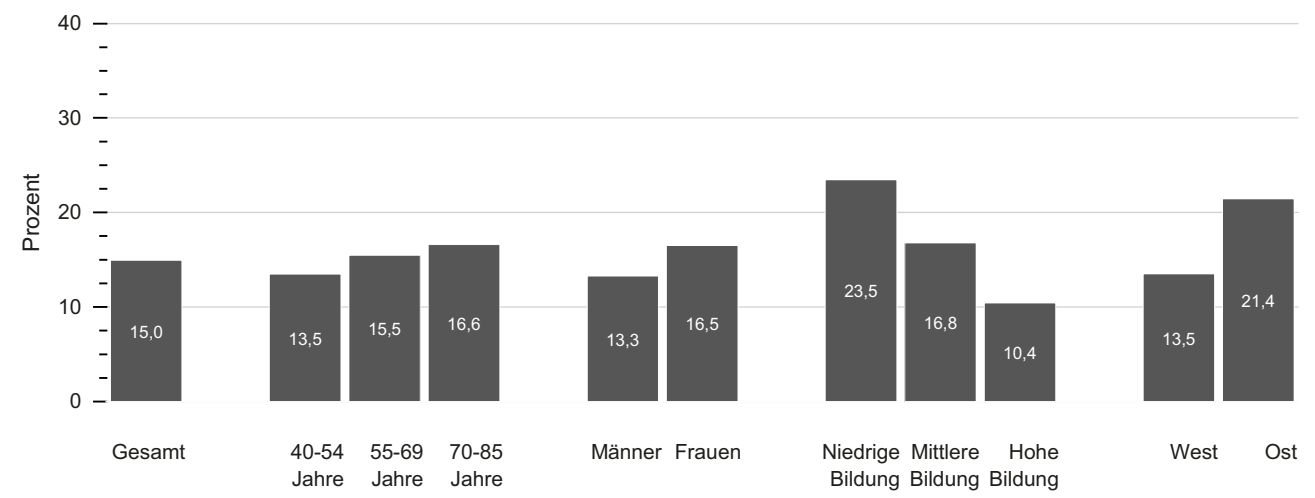

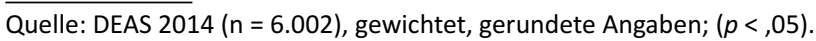

Altersunterschiede nur signifikant zwischen jüngster und ältester Altersgruppe. Signifikante Unterschiede für Geschlecht, Bildung und Landesteil.

Bei den höheren Altersgruppen sind die subjektiven Armutsquoten höher als in den jüngeren Altersgruppen, sodass ein signifikanter Unterschied zwischen der jüngsten und der ältesten Altersgruppe zu finden ist. Im Vergleich dazu sind bei den objektiven Armutsquoten keine signifikanten Unterschiede zwischen den Altersgruppen zu beobachten. Dieses Ergeb- nis zeigt, dass die subjektive Wahrnehmung von Armut, das heißt, die Einschätzung, nicht ausreichend finanziell ausgestattet $\mathrm{zu}$ sein, mit dem Alter variiert. Obwohl nach den objektiven Armutsindikatoren die Ältesten (70 bis 85 Jahre) nicht schlechter gestellt sind als die Jüngsten (40 bis 54 Jahre), entspricht ihr Einkommen häufiger nicht dem, was sie subjektiv 
benötigen, um finanziell zurecht zu kommen. Das kann an Differenzen in der Ausgabenhöhe oder auch an der Erwartung einer Ausgabensteigerung aufgrund von (zukünftiger) Pflegebedürftigkeit liegen.

Wir beobachten die gleichen Geschlechtereffekte bei der subjektiven Armut wie bei der objektiven Armut. Frauen fühlen sich öfter arm als Männer und für beide Gruppen ist der Anteil derer, die sich subjektiv arm fühlen, höher als die objektive Armutsquote. Im Hinblick auf regionale Unterschiede sind, analog zur objektiven Armut, die subjektiven Armutsquoten höher in Ost- als in Westdeutschland.

\section{Die Einschätzung der eigenen finanziellen Situation unterscheidet sich zwischen Bildungsgruppen.}

Besonders interessant ist das Ergebnis für die Bildungsgruppen. Ähnlich wie bei den objektiven Armutsquoten berichten niedriggebildete Personen häufiger als hochgebildete Personen subjektive Armut, das heißt, dass sie nicht genug Geld haben, um finanziell zurecht zu kommen (Abbildung 6-7). Allerdings variieren die Differenzen zwischen objektiven und subjektiven Armutsquoten zwischen den
Bildungsgruppen. Während nach der objektiven Armutsdefinition nur 4,5 Prozent der hochgebildeten Personen arm sind, berichten 10,4 Prozent dieser Bildungsgruppe, dass sie weniger Geld haben als sie für ihren Haushalt für erforderlich halten. Bei den niedriggebildeten Personen ist das Gegenteil zu beobachten. So ist die subjektive Armutsquote mit 23,5 Prozent niedriger als die objektive Armutsquote (34,8 Prozent). Dies bedeutet, dass ein großer Teil der Personen, die objektiv als arm bezeichnet werden, sich selbst nicht als arm einschätzt. Dies kann möglicherweise mit einer erheblichen Bewältigungsfähigkeit und Resilienz dieser Gruppe erklärt werden, kann aber auch dazu führen, dass notwendige Hilfen nicht in Anspruch genommen werden. Der gegenteilige Befund bei den höher gebildeten Personen - die subjektive Armutsquote ist deutlich höher als die objektive - könnte damit erklärt werden, dass sich die Erwartungen daran, was finanziell notwendig ist, um ,über die Runden zu kommen', zwischen den Bildungsgruppen unterscheidet. So können sich Personen mit höherer Bildung und Einkommen öfter arm fühlen, wenn bestimmte Konsumerwartungen nicht erfüllt sind, die von anderen Bildungsgruppen nicht als relevant betrachtet werden.

Abbildung 6-7: Subjektive und objektive Armutsquoten, nach Bildung, im Jahr 2014 (in Prozent)

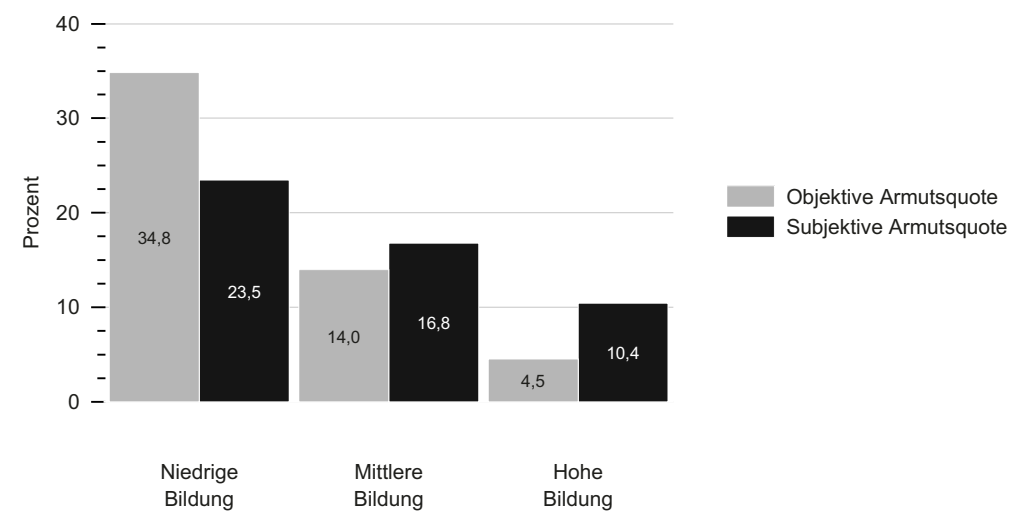

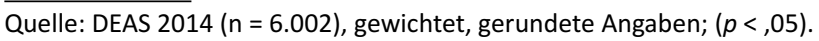

Unterschiede signifikant zwischen die Bildungsgruppen. 


\subsection{Diskussion und Implikationen}

In den letzten Dekaden hat sich der Blick auf die Lebensphase ,Alter' deutlich geändert. Das Alter wird zunehmend auch als Chance und nicht mehr nur als Phase des Verlustes begriffen. In der soziologischen und psychologischen Forschung dominieren Begriffe wie das, gelingende und ,glückliche“ Alter oder auch die ,gebenden', ,produktiven' und ,integrierten' Alten (MotelKlingebiel \& Vogel 2013). Ein Grund für diese positiven Veränderungen der Sichtweise auf das Leben im Alter sind auch die langfristigen Verbesserungen der materiellen Lage Älterer.

Im dritten Altenbericht der Bundesregierung im Jahr 2001 werden ausreichende materielle Ressourcen als Voraussetzung für eine aktive Teilhabe und eine Erweiterung des Interessenspektrums identifiziert (Deutscher Bundestag 2001). Die materiellen Voraussetzungen für die gesellschaftliche Teilhabe sind allerdings nicht für alle Personengruppen gleichermaßen gegeben. Insgesamt ist die Einkommensungleichheit in der zweiten Lebenshälfte im hier beobachteten Zeitraum gestiegen, das heißt, die Voraussetzungen für ein aktives und integriertes Leben im Alter sind zunehmend ungleich verteilt. Bemerkenswert ist in diesem Zusammenhang auch, dass sich hinsichtlich der Einkommen, aber auch hinsichtlich der Armutsquoten im Zeitraum zwischen 1996 und 2014 keine Angleichung zwischen Ost- und Westdeutschland erkennen lässt.

In den hier untersuchten Perioden sind ältere Menschen nicht überdurchschnittlich häufig von Armut betroffen, jedoch ist zukünftig mit einem Anstieg der Armutsgefährdung für Personen im Rentenbezug zu rechnen. Die sozialen Sicherungssysteme wurden seit Ende der 1990er Jahre zurückgebaut. Im Gegenzug wird die private Vorsorge zunehmend wichtiger bei der Sicherung des Lebensstandards im Alter. Darüber hinaus sind Erwerbsverläufe heute oft diskontinuierlicher als früher, was sich voraussichtlich ebenfalls in zukünftig niedrigeren Alterseinkommen niederschlagen wird.

Darüber hinaus gibt es Gruppen, die jetzt schon häufiger armutsgefährdet sind als andere. Insbesondere Frauen sind stark von Altersarmut betroffen. Sie haben häufig zu geringe Rentenanwartschaften aus eigener Erwerbstätigkeit erworben und können damit ihren Lebensstandard im Alter nicht sichern. Fällt das Einkommen des Mannes weg oder hat dieser auch selbst keine ausreichende Rente, leben sie vergleichsweise häufig in Armut. Durch die steigende Erwerbspartizipation von Frauen werden sich ihre Rentenanwartschaften in der Zukunft verbessern. Allerdings werden durch die steigende geringfügige und Teilzeit-Beschäftigung besonders bei Frauen diese Zuwächse nur gering ausfallen und Unterschiede zwischen den Geschlechtern weiterhin existieren.

Des Weiteren sind Personen in Ostdeutschland öfter armutsgefährdet als Personen in Westdeutschland. Ungefähr ein Drittel der Personen mit niedriger Bildung ist ebenfalls armutsgefährdet und der Abstand zwischen hoch- und niedriggebildeten Personen ist seit 2002 stark gestiegen.

Individuell betrachtet ist Altersarmut häufig eine Lebenssituation, die nicht aus eigener Kraft verändert werden kann, da es keinen oder nur einen sehr eingeschränkten Zugang zum Arbeitsmarkt gibt. Dies gilt in besonderem Maße für Personen, die sich bereits im Ruhestandsalter befinden. Die subjektive Bewertung der eigenen Lebenssituation ergänzt die Interpretation der Betroffenheit von Armut und erweitert das Verständnis für diese Lebenslage. Die objektiven Befunde werden im Lichte der subjektiven Bewertung greifbarer und spiegeln die Auswirkungen von Armut wider. Unsere Ergebnisse zeigen, dass wir in den Gruppen, die objektiv häufiger von Armut gefährdet sind, auch höhere Anteile subjektiver Armut finden. Das trifft für Frauen, Menschen in Ostdeutschland sowie für Personen mit niedriger Bildung zu. Dabei sind die subjektiven Armutsquoten höher als die objektiven Armutsquoten (mit der Ausnahme von niedriggebildeten Personen). Schließlich unterscheidet sich die Einschätzung der eigenen finanziellen Situation deutlich zwischen Bildungsgruppen. Bei den unteren Bildungsgruppen gibt es einen hohen Anteil von Menschen, die sich unter der Armutsgrenze befinden, aber sich nicht selbst als arm einschätzen. Diese 
Gruppe ist für die Sozialpolitik von besonderem Interesse, da die Gefahr besteht, dass notwendige politische Maßnahmen aufgrund der nicht sichtbaren negativen Position nicht durchgeführt werden (Zapf 1984).

Das Wissen um die materielle Situation und ihre subjektive Bewertung kann helfen, Bedürftigkeit besser zu verstehen und dementsprechend gegenzusteuern. Nur mit dem Wissen um Risiko- gruppen und spezifische Probleme einzelner Bevölkerungsgruppen können politische Entscheidungen getroffen werden. Vor dem Hintergrund der berichteten Befunde scheint es sinnvoll, armutsvermeidende Maßnahmen insbesondere mit Blick auf Frauen, Personen mit niedriger Bildung sowie Menschen in Ostdeutschland in der zweiten Lebenshälfte zu verstärken.

Altern im sozialen Wandel: Die Rückkehr der Altersarmut? (S. 273-290). Wiesbaden: Springer VS.

Simonson, J., Kelle, N., Romeu Gordo, L., Grabka, M. M., Rasner, A., \& Westermeier, C. (2012). Ostdeutsche Männer um 50 müssen mit geringeren Renten rechnen. DIW Wochenbericht, 23, 3-13.

Statistische Ämter des Bundes und der Länder (2015). Sozialberichterstattung der amtlichen Statistik. Online: http://www.amtliche-sozialberichterstattung.de/ Tabellen_Excel/A1.1.0\%20DE_Bund.xlsx (zuletzt abgerufen 15.07.2016).

Statistisches Bundesamt (2015a). Mikrozensus 2014 Sonderauswertungen für das Deutsche Zentrum für Altersfragen. Wiesbaden: Statistisches Bundesamt.

Statistisches Bundesamt (2015b). OECD-Skala. Online: https://www.destatis.de/DE/ZahlenFakten/GesellschaftStaat/Soziales/Sozialberichterstattung/Glossar/ OECDSkala.html (zuletzt abgerufen 15.07.2016).

Motel-Klingebiel, A., \& Vogel, C. (2013). Altersarmut und die Lebensphase Alter. In: C. Vogel \& A. Motel-Klingebiel (Hrsg.) Altern im sozialen Wandel: Die Rückkehr der Altersarmut? Wiesbaden: Springer VS.

Noll, H.-H., \& Weick, S. (2013). Materieller Lebensstandard im Alter. In: C. Vogel \& A. Motel-Klingebiel (Hrsg.) Altern im sozialen Wandel: Die Rückkehr der Altersarmut? (S. 113-140). Wiesbaden: Springer VS.

Ravallion, M. (2012). Poor, or Just Feeling Poor? On Using Subjective Data in Measuring. Poverty Policy Research Working Paper Series, 5968. Washington D.C.: The World Bank.

Schröder, C. (2013). Armut in Europa [IW-Trends 1/2013]. Köln: Institut der deutschen Wirtschaft.

Simonson, J. (2013). Erwerbsverläufe im Wandel - Konsequenzen und Risiken für die Alterssicherung der Babyboomer. In: C. Vogel \& A. Motel-Klingebiel (Hrsg.)
Vogel, C., \& Künemund, H. (2014). Armut im Alter. In: S. Frech \& O. Groh-Samberg (Hrsg.) Armut in Wohlstandsgesellschaften (S. 123-136). Schwalbach im Taunus: Wochenschau Verlag.

Wetzstein, M., Rommel, A., \& Lange, C. (2015). Pflegende Angehörige - Deutschlands größter Pflegedienst. GBE Kompakt, 6(3), 3-11.

Zapf, W. (1984). Individuelle Wohlfahrt: Lebensbedingungen und wahrgenommene Lebensqualität. In: W. Glatzer \& W. Zapf (Hrsg.) Lebensqualität in der Bundesrepublik. Objektive Lebensbedingungen und subjektives Wohlbefinden (S. 13-26). Frankfurt am Main/New York: Campus Verlag.

Zhu, L. Y., Weißenborn, A., \& Buscher, H. S. (2011). Im Fokus: Altersarmut und soziales Befinden in Ost- und Westdeutschland, 1995 und 2009. Wirtschaft im Wandel, $17(7-8), 240-244$.

Open Access Dieses Kapitel wird unter der Creative Commons Namensnennung 2.5 International Lizenz (http://creativecommons.org/licenses/by/2.5/deed.de) veröffentlicht, welche die Nutzung, Vervielfältigung, Bearbeitung, Verbreitung und Wiedergabe in jeglichem Medium und Format erlaubt, sofern Sie den/die ursprünglichen Autor(en) und die Quelle ordnungsgemäß nennen, einen Link zur Creative Commons Lizenz beifügen und angeben, ob Änderungen vorgenommen wurden.

Die in diesem Kapitel enthaltenen Bilder und sonstiges Drittmaterial unterliegen ebenfalls der genannten Creative Commons Lizenz, sofern sich aus der Abbildungslegende nichts anderes ergibt. Sofern das betreffende Material nicht unter der genannten Creative Commons Lizenz steht und die betreffende Handlung nicht nach gesetzlichen Vorschriften erlaubt ist, ist für die oben aufgeführten Weiterverwendungen des Materials die Einwilligung des jeweiligen Rechteinhabers einzuholen. 